\title{
Optimized Domain Decomposition Method for Non Linear Reaction Advection Diffusion Equation
}

\author{
M.R.Amattouch, PhD \\ N.Nagid, PhD \\ H.Belhadj, Prof. \\ University of Abdelmalek Essaadi, Faculty Of Sciences And Techniques, \\ Department Of Mathematics, Tangier, Morocco
}

doi: 10.19044/esj.2016.v12n27p63 URL:http://dx.doi.org/10.19044/esj.2016.v12n27p63

\begin{abstract}
This work is devoted to an optimized domain decomposition method applied to a non linear reaction advection diffusion equation. The proposed method is based on the idea of the optimized of two order (OO2) method developed this last two decades. We first treat a modified fixed point technique to linearize the problem and then we generalize the $\mathrm{OO} 2$ method and modify it to obtain a new more optimized rate of convergence of the Schwarz algorithm. To compute the new rate of convergence we have used Fourier analysis. For the numerical computation we minimize this rate of convergence using a global optimization algorithm. Several test-cases of analytical problems illustrate this approach and show the efficiency of the proposed new method.
\end{abstract}

Keywords: Non linear reaction advection diffusion equation, Modified fixed point method, Domain decomposition method, Optimized interface conditions, Finite volume discretization

\section{Introduction}

The aim goal of this paper is to propose an optimized domain decomposition method (DDM) to solve a non linear reaction advection diffusion equation on a bounded domain such that:

$$
\left\{\begin{array}{lr}
F(u)+c u+a \frac{\partial u}{\partial x}+b \frac{\partial u}{\partial y}-v \Delta u=f & \text { on } \Omega \\
u=g & \text { on } \partial \Omega
\end{array}\right.
$$

This kind of equations has been treated using domain decomposition methods by many autors these last two decades $[1,2,4,5]$. In the proposed work in $[4,5]$, the autors have developed a new method based on DDM for 
witch they proposed an approximated convergence rate corresponding to an optimized $\mathrm{OO} 2$ interface conditions. The difficulty of this method is that the rate of convergence is not given explicitly, and its approximation is not given precisely.

In the article [3] we have developed more faster algorithm of Newton-Krylov-Schwarz applied to non stationary problem. Several authors have used many other ideas. For example in [8,9] we propose Newton or quasi-Newton type methods to solve the non linear system such that Broyden-Fletcher-Goldfarb-Shanno (BFGS) method.

These kinds of Newton methods can be used in the context of DDM, but numerically the convergence takes a long time for many non-linearity test-cases.

In the present work we suggest a new approach based on the fixed point theorem to solve the nonlinear partial differential equation of reactionadvection-diffusion that we meet most time in the fluid dynamics and environmental problems.

We propose to use the Schauder fixed point theorem to linearize the two sub-problems. We have calculated explicitly the rate of the optimized two order DDM using this technique and Fourier analysis. It is easy to optimize the rate of convergence using global optimization functions that are implemented in different softwares (Scilab, Matlab,..).

In this article we first present the method based on the fixed point theorem for which we prove the convergence and we show some numerical results obtained using the Scilab software.

In the second part of this work, we present the decomposition domain optimized order two suggested in $[1,2]$ and we develop the technique for calculating explicitly the rate of convergence.

Several numerical examples show the efficiency of the proposed method.

\section{The Modified Fixed point method}

In this section we treat the stationary nonlinear reaction advection diffusion problem:

$$
\left\{\begin{array}{lr}
F(u)+c u+a \frac{\partial u}{\partial x}+b \frac{\partial u}{\partial y}-v \Delta u=f & \text { on } \Omega \\
u=0 & \text { on } \partial \Omega
\end{array}\right.
$$

For a given one initial function $u_{0}$, we construct a sequence of functions $u_{n}$ such that: 


$$
\left\{\begin{array}{cc}
c u_{n+1}+a \frac{\partial u_{n+1}}{\partial x}+b \frac{\partial u_{n+1}}{\partial y}-v \Delta u_{n+1}=f-F\left(u_{n}\right) & \text { on } \Omega \\
u_{n+1}=0 & \text { on } \partial \Omega
\end{array}\right.
$$

In the following we show the convergence of $u_{n}$ to the solution of problem (2).

Let $V=H_{0}^{1}(\Omega)$. Consider the application

$\varphi: V \rightarrow V$

$v \mapsto u$

such that $\mathrm{u}$ is the solution of the variational formulation:

$\int_{\Omega} c u w+\int_{\Omega} \vec{a} \nabla u w+\int_{\Omega} \nabla u \nabla w=\int_{\Omega}(f-F(v)) w \quad \forall w \in V$

where

$\vec{a}=\left(\begin{array}{l}a \\ b\end{array}\right)$

Lemma 1.1 if the assumptions:

- $\exists v \succ 0 ; c-\frac{1}{2} \operatorname{div} \vec{a} \geq 0$; and $v \prec \mu$

- $a, b, c$, and $\mu$ are bounded functions.

are verified, we assume that the variational formulation (4) has an unique solution $u$ in $V$

Proof : Consider the bilinear form $a$ :

$a(u, w)=\int_{\Omega} c u w+\int_{\Omega} \vec{a} \nabla u w+\int_{\Omega} \mu \nabla u \nabla w$

by the Holder and the Poincarré Inequalities $a$ is continuous:

$$
\begin{aligned}
a(u, w) \leq & \left(\sup _{\Omega}|c|\right)\|u\|\|w\|+\sup _{\Omega}\|\vec{a}\| \quad\|\nabla u\| w\left\|+\sup _{\Omega} \mu\right\| \nabla u\|\nabla w\| \\
& \leq\left(\sup _{\Omega}|c|+\sup _{\Omega}\|\vec{a}\| C_{\text {poincarre }}+C_{\text {poincarre }}^{2} \sup _{\Omega} \mu\right)\|\nabla u\| \nabla w \|
\end{aligned}
$$

$a$ is coercive:

$$
\begin{aligned}
\int_{\Omega} \vec{a} \nabla u w & =-\int_{\Omega} u \vec{a} \nabla w-\int_{\Omega} \operatorname{div} \vec{a} u w+\int_{\partial \Omega} \vec{n} \vec{a} u w \\
& =-\int_{\Omega} u \vec{a} \nabla w-\int_{\Omega} \operatorname{div} \vec{a} u w
\end{aligned}
$$

Thus, 


$$
\begin{aligned}
a(u, u) & =\int_{\Omega} c u u+\frac{1}{2} \int_{\Omega} \vec{a} \nabla u u+\frac{1}{2} \int_{\Omega} \vec{a} \nabla u u+\int_{\Omega} \mu \nabla u \nabla u \\
& =\int_{\Omega} c u u-\frac{1}{2} \int_{\Omega} u \vec{a} \nabla u-\frac{1}{2} \int_{\Omega} \operatorname{div} \vec{a} u u+\frac{1}{2} \int_{\Omega} \vec{a} \nabla u u+\int_{\Omega} \mu \nabla u \nabla u \\
& =\int_{\Omega}\left(c-\frac{1}{2} \operatorname{div} \vec{a}\right) u^{2}+\int_{\Omega} \mu\|\nabla u\|^{2} \\
& \geq v\|u\|^{2}
\end{aligned}
$$

The Lax Milgram theorem involves that there exists one solution of

(2) named $\varphi(v)$

we have by substraction:

$$
a(\varphi(u)-\varphi(v), w)=\int_{\Omega}(F(u)-F(v)) w \quad \forall w \in V
$$

Theorem 1.1 Suppose that:

- $\exists v \succ 0 ; c-\frac{1}{2} \operatorname{div} \vec{a} \geq 0$ and $\mu \succ v$

- Fis K-lipshitzian.

- c, a,b and $\mu$ are bounded functions and $\frac{K}{\mu} \prec \prec 1$

Then the sequence $u_{n+1}=\varphi\left(u_{n}\right)$ converges to the unique solution of the nonlinear problem (2)

Proof. The application $\varphi$ bellow is well defined by lemma 1.1

We take $w=\varphi(u)-\varphi(v)$ used in the coercivity of $a(.,$.$) in the proof of the$ lemma 1.1 then

$$
\begin{gathered}
a(\varphi(u)-\varphi(v), \varphi(u)-\varphi(v))=\int_{\Omega}(F(u)-F(v))(\varphi(u)-\varphi(v)) \\
\geq v\|\varphi(u)-\varphi(v)\|^{2}
\end{gathered}
$$

$F$ is K-lipshitzian, then we have:

$$
v\|\varphi(u)-\varphi(v)\|^{2} \leq K\|u-v\|\|\varphi(u)-\varphi(v)\|
$$

$$
\frac{K}{v} \prec \prec 1 \text { then } \varphi \text { is a contraction, and so, applying the Shauder fixed point }
$$

theorem to the application $\varphi$ we show that the equation $\varphi(u)=u)$ have one solution and the sequence $u_{n+1}=\varphi\left(u_{n}\right)$ converges to this solution which is the solution of problem (3). 
In the proof of the theorem we saw that:

$v|\varphi(u)-\varphi(v)|^{2} \leq K|u-v||\varphi(u)-\varphi(v)|$

The convergence of the the fixed point algorithm is faster when $\mathrm{K}$ is increasingly small, so for obtaining a small coefficient $\mathrm{K}$ we propose to modify the problem (3) as follows:

$$
\left\{\begin{array}{c}
(c-\xi) u_{n+1}+\vec{a} \nabla u_{n+1}-\mu \Delta u_{n+1}=f-F\left(u_{n}\right)-\xi u_{n} \quad \text { on } \Omega \\
u_{n+1}=0 \text { on } \partial \Omega
\end{array}\right.
$$

The parameter $\xi$ is added to the system (3) to construct a new sequence with a smaller Lipshitzian K coefficient.

Theorem 1.2 Suppose that:

- $\exists v \succ 0 ; c-\frac{1}{2} \operatorname{div} \vec{a} \geq 0$ and $\mu \succ v$

- $c, a, b$ and $\mu$ are bounded

- $F \in C^{2}(R)$

- $\xi(x)=F^{\prime}(|x|)$

Then the sequence $u_{n}$ of problem (3) converges to the solution of the nonlinear problem (1).

Proof. We assume that there exists a $\varepsilon<1$ such that

$\left.\left|F(z)-F(y)-F^{\prime}(|x|)(z-y)\right| \prec \varepsilon|z-y| \quad \forall y, z \in\right] x-\varepsilon, x+\varepsilon\left[\quad\left(F \in C^{2}(R)\right)\right.$

Consider $V=\left\{u \in H_{0}^{1}(\Omega) / u-|x|_{\infty} \leq \varepsilon\right\}$ and let

$$
\begin{gathered}
\varphi: V \rightarrow V \\
v \mapsto u
\end{gathered}
$$

such that $\mathrm{u}$ is the solution of the variational formulation:

$\int_{\Omega}\left(c+F^{\prime}(\mid x)\right) u w+\int_{\Omega} \vec{a} \nabla u w+\int_{\Omega} \mu \nabla u \nabla w=\int_{\Omega}\left(f+F^{\prime}(|x|) v-F(v)\right) w \forall w \in H_{0}^{1}(\Omega)$

As we have done for the proof of Theorem 1.1, we have: $\varphi$ is well defined because of theorem 1.3 and we show that:

$$
\begin{aligned}
a(\varphi(u)-\varphi(v), \varphi(u)-\varphi(v))=\int_{\Omega} & \left(F(u)-F(v)-F^{\prime}(\mid x)(u-v)\right)(\varphi(u)-\varphi(v)) \\
& \geq v\|\varphi(u)-\varphi(v)\|^{2}
\end{aligned}
$$

Thus

$v|\varphi(u)-\varphi(v)|^{2} \leq \varepsilon|u-v||\varphi(u)-\varphi(v)|$ 
and then $\varphi$ is continuous, so the Schauder theorem involves that the sequence $u_{n}$ of problem (3) converges to the unique solution of the weak formulation of the nonlinear problem (1) $\square$

In the next theorem we generalize the lemma (1.1) for all functions a, $\mathrm{b}$ and $\mathrm{c}$ for the linear problem:

$$
\left\{\begin{array}{c}
c u+\vec{a} \nabla u-\mu \Delta u=f \quad \text { on } \Omega \\
u=0 \quad \text { on } \partial \Omega
\end{array}\right.
$$

Notice that the first condition of lemma (1.1) is omitted in the next theorem.

\section{Theorem 0.3 Suppose that:}

- $\exists v>0 ; \mu(x) \geq v \quad \forall x \in \Omega$

- $c, a, b$ and $\mu$ are given arbitrary bounded functions.

Then the problem (4) has an unique solution in $H_{0}^{1}(\Omega)$.

Proof. We can choose function $C_{0}$ and two parameters $\varepsilon$ and $\mu_{0}$ such that:

$-\frac{\mu_{0}}{\varepsilon \mu+\mu_{0}} \prec \frac{1}{2}$

- $C_{0}+\varepsilon\left(c-\frac{1}{2} \operatorname{div} \vec{a}\right) \geq 0$

- $\frac{\varepsilon \mu+\mu_{0}}{2} \geq C_{0} \geq 0$

So consider:

$\varphi: V \rightarrow V$

$$
v \mapsto u
$$

such that $\mathrm{u}$ is the solution of the variational problem:

$$
\begin{gathered}
\int_{\Omega}\left(C_{0}+\varepsilon c\right) u w+\int_{\Omega} \varepsilon \vec{a} \nabla u w+\int_{\Omega}\left(\mu+\varepsilon \mu_{0}\right) \nabla u \nabla w=\int_{\Omega}\left(f+C_{0}\right) v w+ \\
\ldots+\int_{\Omega} \mu_{0} \nabla v \nabla w \quad \forall w \in V
\end{gathered}
$$

As we have done for the proof of Theorem 1.1 we have: $\varphi$ is well defined because

$$
c+C_{0}-\frac{1}{2} \operatorname{div} \vec{a} \geq 0
$$

and we show that:

$$
\begin{gathered}
a(\varphi(u)-\varphi(v), \varphi(u)-\varphi(v))=\int_{\Omega}\left(C_{0}(u-v)(\varphi(u)-\varphi(v))+\ldots\right. \\
\left.\quad \ldots \mu_{0} \int_{\Omega} \nabla(u-v) \nabla(\varphi(u)-\varphi(v))\right) \geq\left(\varepsilon v+\mu_{0}\right)\|\varphi(u)-\varphi(v)\|^{2}
\end{gathered}
$$


Thus

$\left(\varepsilon v+\mu_{0}\right)|\varphi(u)-\varphi(v)|^{2} \leq\left(C_{0}+\mu_{0}\right)|u-v||\varphi(u)-\varphi(v)|$

and then $\varphi$ is continuous, so by the Schauder theorem $\varphi$ has one fixed point which is the solution of problem (4) $\square$

Remark 1 To accelerate the fixed point of problem (1) we propose the sequence $u_{n}$ such that:

$\left\{\begin{array}{c}(c-\xi) u_{n+1}+a \nabla u_{n+1}-\mu \Delta u_{n+1}=f-F\left(u_{n}\right)-\xi u_{n} \text { on } \Omega(9) \\ u_{n+1}=0 \text { on } \partial \Omega\end{array}\right.$

we take for example

$\xi=F^{\prime}(\varphi(x))$

$\xi=F^{\prime}\left(u_{n}\right)$

$\xi=\frac{F\left(u_{n}\right)-F(0)}{u_{n}}$.

Remark 2 With the same way we can apply this concept of fixed point to the general following problem:

$$
\left\{\begin{array}{c}
\frac{\partial u}{\partial t}+F(u)+A(u) \nabla u-\mu(u) \Delta u \quad \text { on } \Omega \times[0, T] \\
u=g \quad \text { on } \partial \Omega \times[0, T] \\
u(t=0)=h
\end{array}\right.
$$

where $A$ and $B$ and $\mu$ are non linear functions. Indeed:

For a given initial function $u_{0}$, we construct iteratively a sequence function $u_{n}$ as follows:

$$
\left\{\begin{array}{c}
\frac{\partial u_{n+1}}{\partial t}+c u_{n+1}+A\left(u_{n}\right) \nabla u_{n+1}-\mu\left(u_{n}\right) \Delta u_{n+1}=f-F\left(u_{n}\right) \quad \text { on } \Omega \times[0, T] \\
u_{n+1}=g \quad \text { on } \partial \Omega \times[0, T] \\
u_{n+1}(t=0)=h
\end{array}\right.
$$

Domain decomposition with optimized interface of two order(DDM OO2):

The use of finite volumes, finite differences or finite elements solvers on high order meshes requires a high cost of computation. 
Domain decomposition methods can reduce this cost by splitting initial problem into two or more sub-problems with smaller dimensions. Many authors have studied domain decomposition methods these last decades $[10,11]$. Among these methods we consider in this work the method called second order optimized method OO2. This method was developed by different authors $[1,2]$. The main idea of the $\mathrm{OO} 2$ technique is described briefly as follows:

We split the domain $\Omega$ in two sub-domains $\Omega_{1}$ and $\Omega_{2}$ with an interface $\Gamma$ (see figure 1)

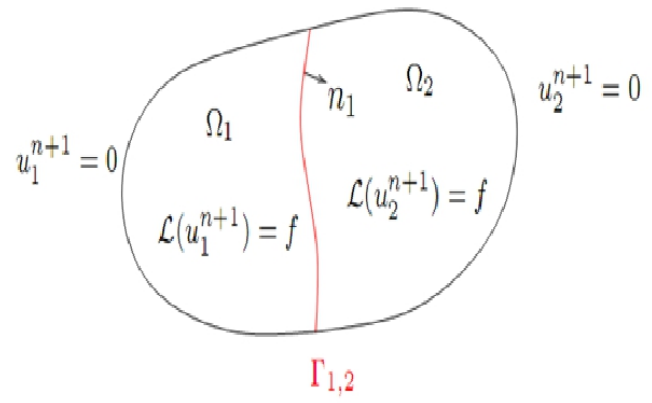

then we built two sequences $u_{1}^{p}$ and $u_{2}^{p}$ respectively solutions of two sub-problems as described bellow:

we choose two initials functions $u_{1}^{0}$ defined on $\Omega_{1}$ and $u_{2}^{0}$ defined on $\Omega_{2}$ then we consider the two problems:

$$
\left\{\begin{array}{c}
L\left(u_{1}^{p+1}\right)=f \quad \text { on } \Omega_{1} \\
u_{1}^{p+1}=g \quad \text { on } \partial \Omega \cap \Omega_{1} \\
B_{1}\left(u_{1}^{p+1}\right)=B_{1}\left(u_{2}^{p}\right) \text { on } \Gamma
\end{array}\right.
$$

And

$$
\left\{\begin{array}{c}
L\left(u_{2}^{p+1}\right)=f \quad \text { on } \Omega_{2} \\
u_{2}^{p+1}=g \quad \text { on } \partial \Omega \cap \Omega_{2} \\
B_{2}\left(u_{2}^{p+1}\right)=B_{2}\left(u_{1}^{p}\right) \text { on } \Gamma
\end{array}\right.
$$

Where

$$
\begin{aligned}
& L(u)=c u+\vec{a} \nabla u-\mu \Delta u \\
& \left\{\begin{array}{c}
B_{1}(u)=\frac{\partial u}{\partial n}-C_{1} u+C_{2} \frac{\partial u}{\partial \tau}-C_{3} \frac{\partial^{2} u}{\partial \tau^{2}} \\
B_{2}(u)=-\frac{\partial u}{\partial n}-\left(C_{1}-\frac{a}{\mu}\right) u+C_{2} \frac{\partial u}{\partial \tau}-C_{3} \frac{\partial^{2} u}{\partial \tau^{2}}
\end{array}\right.
\end{aligned}
$$


$\mathrm{n}$ and $\tau$ are the normal and the tangent on $\Omega_{1}$

Because of the Fourier analysis we show that the rate of convergence in the fourier way is (see [6] for proof)

$$
\rho\left(C_{1}, C_{2}, C_{3}, k\right)=\left(\frac{\lambda^{-}(k)-C_{1}+i k C_{2}+C_{3} k^{2}}{\lambda^{+}(k)+\frac{a}{\mu}-C_{1}+i k C_{2}+C_{3} k^{2}}\right)^{2}
$$

where

$$
\lambda^{ \pm}(k)=\frac{a \pm \sqrt{a^{2}+4 c \mu-4 i k \mu b+4 k^{2} \mu^{2}}}{2 \mu}
$$

In the following we resume the demonstration in the case of which the interface $\Gamma$ is the line $x=0$, then the normal and the tangent vector are respectively $n=\vec{i}$ and $\tau=\vec{j}$

we have by substraction:

$$
\begin{aligned}
& L\left(u_{1}^{p+2}-u\right)=0 \text { and } L\left(u_{2}^{p+1}-u\right)=0 \\
& B_{1}\left(u_{1}^{p+2}-u\right)=B_{1}\left(u_{2}^{p+1}-u\right) ; \text { and } B_{2}\left(u_{2}^{p+1}-u\right)=B_{2}\left(u_{1}^{p}-u\right)
\end{aligned}
$$

$\mathrm{u}$ is the exact solution of the problem . if $\omega^{p+2}$ is the fourier transform of $u_{1}^{p+2}-u$, we have

$c \omega^{p+2}+a \frac{\partial \omega^{p+2}}{\partial x}-i b k \omega^{p+2}-\mu \frac{\partial^{2} \omega^{p+2}}{\partial x^{2}}+k^{2} \omega^{p+2}=0$

$\mathrm{k}$ is the fourier frequency. If we take a solution in the form of $\omega(0, k) e^{\lambda x}$ we have

$c+a \lambda-i b k-\mu \lambda^{2}+\mu k^{2}=0$

it's an equation of two order which have two solution

$$
\lambda^{ \pm}(k)=\frac{a \pm \sqrt{a^{2}+4 c \mu-4 i k \mu b+4 k^{2} \mu^{2}}}{2 \mu}
$$

since $\lim _{x \rightarrow-\infty} \omega^{p+2}(x, k)=0$ we take $\omega^{p+2}(x, k)=\omega^{p+2}(0, k) e^{\lambda^{+} x}$ (remark that $\lambda^{+} \succ 0 \quad$ and $\left.\quad \lambda^{-} \prec 0\right)$

by the same way if $\omega^{p+1}$ is the fourier transform of $\boldsymbol{u}_{2}^{p+1}-\boldsymbol{u}$, we have

$$
\omega^{p+1}(x, k)=\omega^{p+1}(0, k) e^{\lambda^{-} x}
$$

The conditions 
$B_{1}\left(\omega^{p+2}(0, k)\right)=B_{1}\left(\omega^{p+1}(0, k)\right)$ and $B_{2}\left(\omega^{p+1}(0, k)\right)=B_{2}\left(\omega^{p}(0, k)\right)$ implies

$$
\begin{aligned}
& \left(\lambda^{+}(k)-C_{1}+i k C_{2}+C_{3} k^{2}\right) \omega^{p+2}(0, k)=\left(\lambda^{-}(k)-C_{1}+i k C_{2}+C_{3} k^{2}\right) \omega^{p+1}(0, k) \\
& \left(\lambda^{-}(k)-C_{1}+\frac{a}{\mu}+i k C_{2}+C_{3} k^{2}\right) \omega^{p+1}(0, k)=\left(\lambda^{+}(k)-C_{1}+\frac{a}{\mu}+i k C_{2}+C_{3} k^{2}\right) \omega^{p}(0, k)
\end{aligned}
$$

Thus the rate of convergence is

$$
\rho\left(C_{1}, C_{2}, C_{3}, k\right)=\frac{\omega^{p+2}(0, k)}{\omega^{p}(0, k)}=\frac{\lambda^{-}(k)-C_{1}+i k C_{2}+C_{3} k^{2}}{\lambda^{+}(k)-C_{1}+i k C_{2}+C_{3} k^{2}} \times \frac{\lambda^{+}(k)-C_{1}+\frac{a}{\mu}+i k C_{2}+C_{3} k^{2}}{\lambda^{-}(k)-C_{1}+\frac{a}{\mu}+i k C_{2}+C_{3} k^{2}}
$$

Since $\lambda^{+}+\lambda^{-}=\frac{a}{\mu}$, we have expression (11) The next theorem show the convergence of $\mathrm{OO} 2$ method as developed in [6]

Theorem 2.4 Suppose that $c>0$ and $\operatorname{sign}(b)=\operatorname{sign}\left(C_{2}\right)$ and $C_{3} \geq 0$ then,

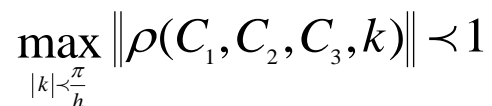

Proof. see [6]

Remark 3 In order to optimize the method we need to optimize the rate of convergence so we look for:

$$
\min _{C_{1}, C_{2}, C_{3}} \max _{|k|<\frac{\pi}{h}}\left\|\rho\left(C_{1}, C_{2}, C_{3}, k\right)\right\| \prec 1
$$

For optimizing this rate we have implemented the global optimization method [7]. Notice that this last problem have at least two optimums.

Remark 4 The OO2 method converges if $c>0$. It can be generalized to a problem with coefficients $a$ and $b$ are variables. Nevertheless, if $c$ is negative the method is not convergent in the most cases of this kind of equations.

In the next theorem we prove the convergence this problem when $c<0$ :

Theorem 0.5 Let $c<0$ then the problem (12) and (13) converge when applying the 002 method

Proof. If $c<0$ then $1-c>0$, so applying the following fixed point

$$
\left\{\begin{array}{c}
(1-c) u_{n+1}+\vec{a} \nabla u_{n+1}-\mu \Delta u_{n+1}=f-F\left(u_{n}\right)-(2 c-1) u_{n} \\
u_{n+1}=g \text { on } \partial \Omega
\end{array}\right.
$$


the $\mathrm{OO} 2$ algorithm converges because $1-c>0$. $\square$

We take the generalized artificial coefficients:

$\left\{\begin{array}{c}B_{1}^{\prime}(u)=\frac{\partial u}{\partial n}-C_{1} u+C_{2} \frac{\partial u}{\partial \tau}-C_{3} \frac{\partial^{2} u}{\partial \tau^{2}}+\frac{\partial u}{\partial n}-C_{1} u(0, y-a)+C_{2} \frac{\partial u(0, y-a)}{\partial \tau}-C_{3} \frac{\partial^{2} u(y-a)}{\partial \tau^{2}} \\ B_{2}(u)=-\frac{\partial u}{\partial n}-\left(C_{1}-\frac{a}{\mu}\right) u+C_{2} \frac{\partial u}{\partial \tau}-C_{3} \frac{\partial^{2} u}{\partial \tau^{2}}-\left(C_{1}-\frac{a}{\mu}\right) u(0, y+a)+C_{2} \frac{\partial u(0, y+a)}{\partial \tau}-C_{3} \frac{\partial^{2} u(y+a)}{\partial \tau^{2}}\end{array}\right.$

The Fourier transform of the the rate of convergence is calculated using:

$F(f(y-a))=\exp i k a F(f)$

The Coupled $\mathrm{OO} 2$ and Fixed point leads to the next subproblems:

$$
\left\{\begin{array}{c}
L\left(u_{1}^{p+1}\right)=f-F\left(u_{1}^{p}\right)-\xi u_{1}^{p} \text { on } \Omega_{1} \\
u_{1}^{p+1}=g \text { on } \partial \Omega \cap \Omega_{1} \\
B_{1}\left(u_{1}^{p+1}\right)=B_{1}\left(u_{2}^{p}\right) \text { on } \Gamma
\end{array}\right.
$$

and

$$
\left\{\begin{array}{c}
L\left(u_{2}^{p+1}\right)=f-F\left(u_{2}^{p}\right)-\xi u_{2}^{p} \text { on } \Omega_{2} \\
u_{2}^{p+1}=g \quad \text { on } \partial \Omega \cap \Omega_{2} \\
B_{2}\left(u_{2}^{p+1}\right)=B_{2}\left(u_{1}^{p}\right) \text { on } \Gamma
\end{array}\right.
$$

where:

$$
L(u)=(c-\xi) u+\vec{a} \nabla u-\mu \Delta u
$$

Remark 5 For the method consisting in the resolution of the partial differential equation (1.1) by the finite volumes method, or finite differences method and solving the obtained non linear system using Newton, the convergence rate depends on the solution of partial differential equation. So it's impossible to compute this rate of convergence. One idea is to suggest a non linear decomposition [4,5] by eliminating the terms that we don't know in the convergence rate. Unfortunately, this method is often inaccurate.

\section{Finite volume discretization:}

To apply the finite volumes method, we choose a squared control volume C (figure (2)) 


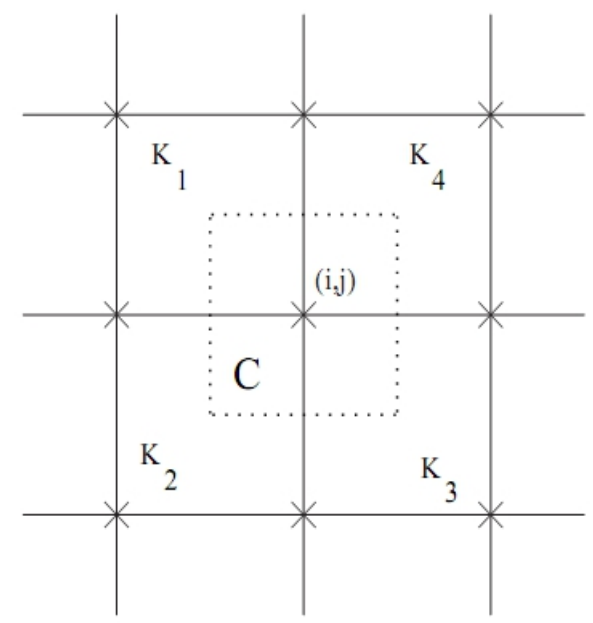

Figure 2: Control volume

and we integer partial differential equation on $\mathrm{C}$ :

$$
\int_{C} c u+\int_{C} a \frac{\partial u}{\partial x}+\int_{C} b \frac{\partial u}{\partial y}-\int_{C} \mu \Delta u=\int_{C} f
$$

We approximate each member of this equation(using classical Finite volume method):

$$
\begin{aligned}
& \int_{C} c u \approx \frac{u_{i ; j} c_{i ; j}}{4} \sum_{l=1}^{4} \operatorname{Vol}\left(K_{l}\right) \\
& \int_{C} \vec{a} \nabla u \approx \frac{1}{4} \sum_{l=1}^{4} \int_{K_{l}} \vec{a} \nabla u \approx \frac{1}{4} \sum_{l=1}^{4} \int_{\partial K_{l}} \vec{a} \vec{n} u \\
& \int_{C} \mu \Delta u=\int_{\partial C} \mu \frac{\partial u}{\partial n}
\end{aligned}
$$

if the control volume $C$ is on the interface $\Gamma$ (figure (3)), we integer the partial differential equation on both $C \cup \Omega_{1}$ and $C \cup \Omega_{2}$. 
$\Omega_{1}$

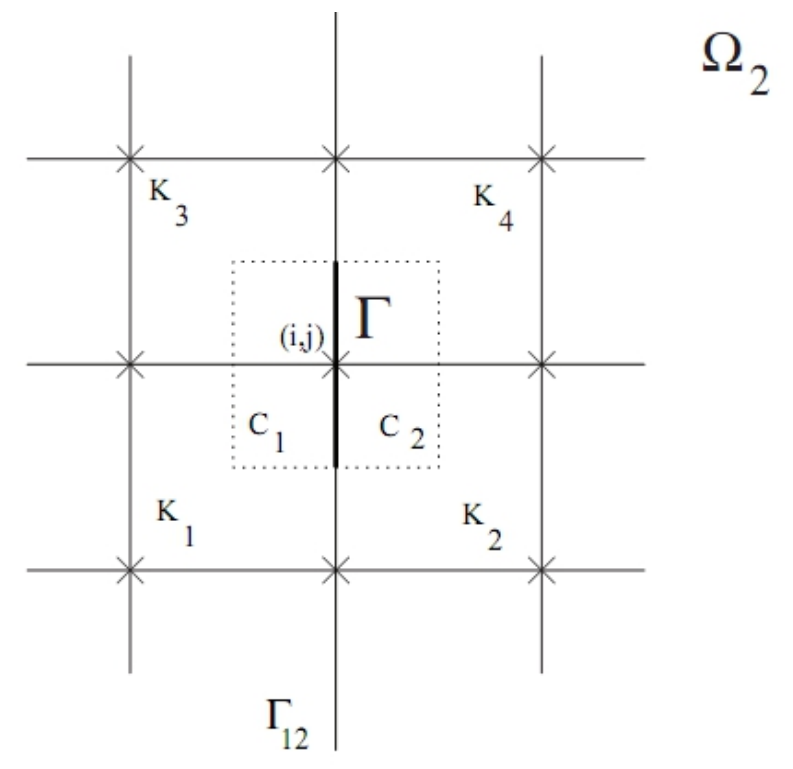

Figure 3: Control volume which include a part of the interface

So we have at iteration $\mathrm{p}+1$ of the method (7) and (8):

$\int_{C_{1}} L\left(u_{1}^{p+1}\right)+\int_{C_{2}} L\left(u_{2}^{p}\right)=\int_{C_{1}} f+\int_{C_{2}} f$

where,

$\int_{C_{i}} L(u)=\int_{C_{i}} c u+\int_{C_{i}} \vec{a} \nabla u-\int_{C_{i}} \mu \Delta u$

the terms $\int_{C_{i}} c u+\int_{C_{i}} \vec{a} \nabla u$ are calculated as presented before. and we have:

$\int_{C_{i}} \mu \Delta u=\int_{C_{i} / \Gamma} \mu \frac{\partial u}{\partial n_{i}}+\int_{\Gamma} \mu \frac{\partial u}{\partial n_{i}}$

Replacing in (12):

$$
\begin{gathered}
\int_{C_{1}} c u_{1}^{p+1}+\int_{C_{1}} \vec{a} \nabla u_{1}^{p+1}+\int_{C_{2}} c u_{2}^{p}+\int_{C_{2}} \vec{a} \nabla u_{2}^{p}+\int_{C_{1} / \Gamma} \mu \frac{\partial u_{1}^{p+1}}{\partial n_{1}}+\int_{C_{2} / \Gamma} \mu \frac{\partial u_{2}^{p}}{\partial n_{2}}+ \\
\ldots+\int_{\Gamma} \mu\left(\frac{\partial u_{1}^{p+1}}{\partial n_{1}}-\frac{\partial u_{2}^{p}}{\partial n_{2}}\right)=\int_{C_{1}} f+\int_{C_{2}} f
\end{gathered}
$$

because $B_{1}\left(u_{1}^{p+1}\right)=B_{1}\left(u_{2}^{p}\right)$ we have: 
$\frac{\partial u_{1}^{p+1}}{\partial n_{1}}-\frac{\partial u_{2}^{p}}{\partial n_{2}}=\left(C_{1}-C_{2} \frac{\partial}{\partial \tau_{1}}+C_{3} \frac{\partial^{2}}{\partial \tau_{1}^{2}}\right)\left(u_{1}^{p+1}-u_{2}^{p}\right)$

so we replace this equality in the last equation to complete the interface condition and to ensure the convergence of the finite volumes method.

\section{Numerical simulation}

Numerically, we have implemented the finite volumes method to approach the sub-problems obtained after applying the fixed point algorithm.

We obtain a good accuracy, the error is in the order of $10^{-5}$ forward the third iteration on some usual test-functions. We take for $\mathrm{F}$ respectively the expressions

$F(u)=u^{p}$

$F(u)=e^{u}$

$F(u)=\frac{a}{b+u}$

$F(u)=\log (1-u)$

The following results show the error between the approximate solution using the modified fixed point method and the exact solution uexact $=x y(1-x)(1-y)$ of the problem (1). Error $=\left\|u-u_{\text {exact }}\right\|_{L^{2}}$

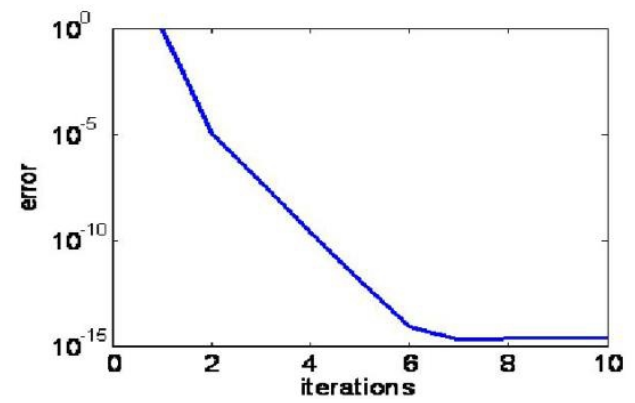

Figure 4: The error: $c=\mu=1, a=b=0 h=0.025 ; F(u)=u * u$

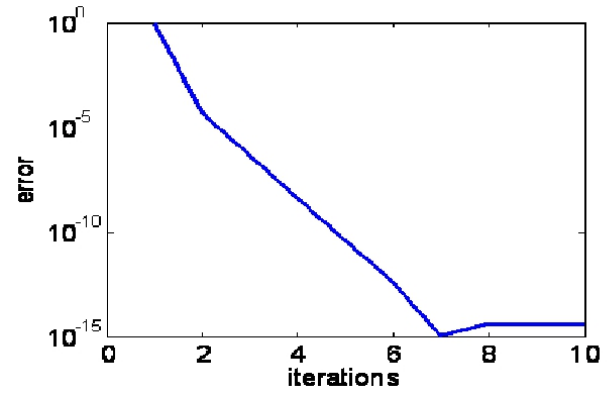

Figure 5: The error: $c=2, \mu=0.1, a=-1, b=0, h=0.001$ and $F(u)=\exp (u)$ 


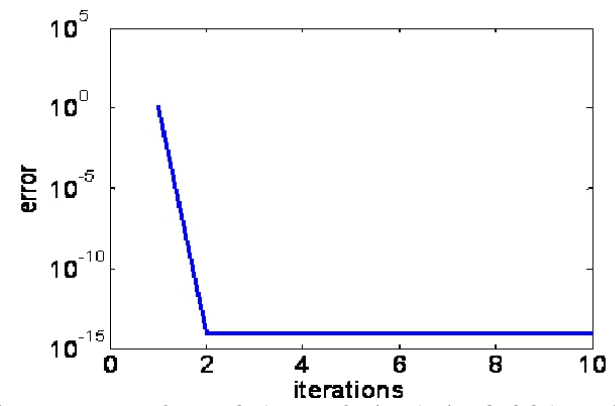

Figure 6: The error: $c=2, \mu=0.1, a=-2, b=1, h=0.001$ and $F(u)=a /(b+u)$

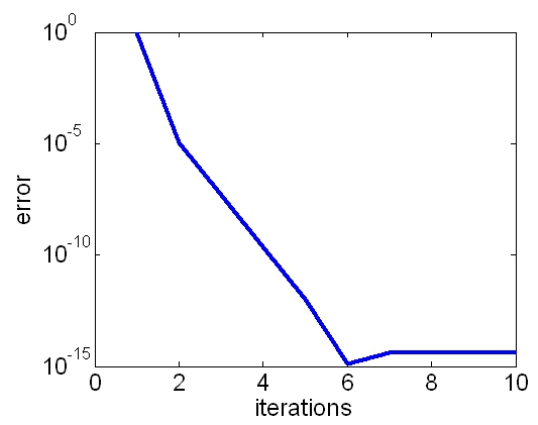

Figure 7: $c=-1, \mu=0.5, a=2, b=-1.5, h=0.001$ and $F(u)=\log (1-u)$

$\mathbf{h}$ is the mesh grid.

Now, we show the results of combining modified fixed point and $\mathrm{OO} 2$ algorithm.

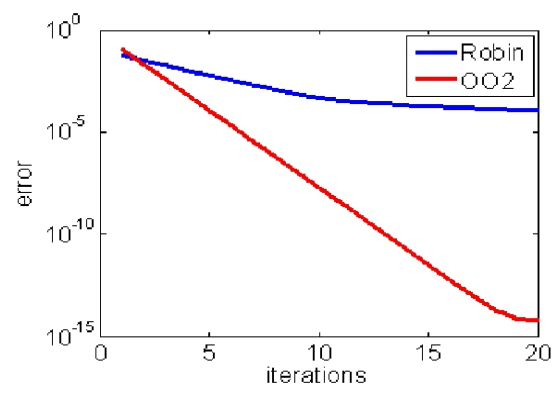

Figure 8: case1

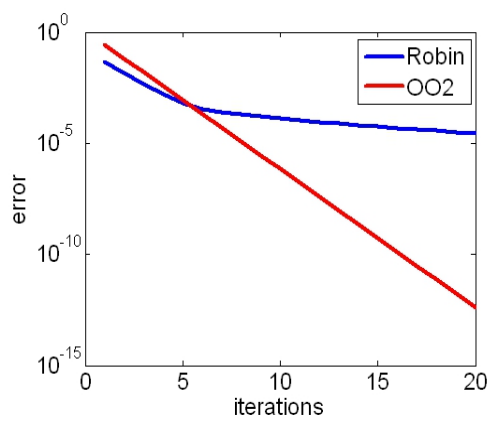

Figure 10: case3

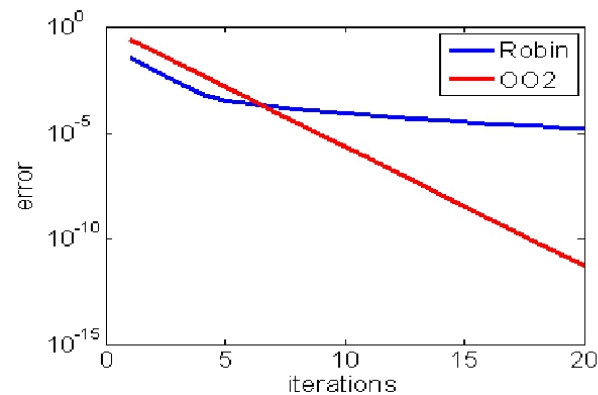

Figure 9: case2

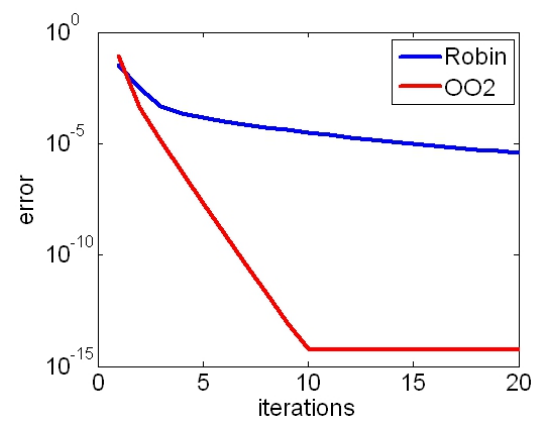

Figure 11: case4 
The figures $8,9,10,11$ showing the $L^{2}$ error between the combining modified fixed point solution and the $\mathrm{OO} 2$ algorithm and the exact solution.

Test case1: $\mathrm{c}=0.1, \quad \mu=1, \quad \mathrm{a}=\mathrm{b}=-2, \quad \mathrm{~h}=0.025, \quad F(u)=\log (u)$ and $u_{\text {exact }}=x y(1-x)(1-y)$.

Test case 2: $\mathrm{c}=2, \quad \mu=0.1, \quad \mathrm{a}=-1, \quad \mathrm{~b}=0, \quad \mathrm{~h}=0.001, \quad \mathrm{~F}(\mathrm{u})=u^{2}$ and uexact $=\exp (x y(1-x)(1-y))-1$.

Test case 3: $\mathrm{c}=-2, \mu=0.1, \mathrm{a}=-2, \mathrm{~b}=1, \mathrm{~h}=0,001, \quad F(u)=u^{3}$ and $u_{\text {exact }}=\exp (x y(1-x)(1-y))-1$.

Test case4: $\mathrm{c}=-1, \quad \mu=0.5 \quad, \quad a=\sin (x y), \quad b=-\cos (x y), \quad \mathrm{h}=0,001$, $F(u)=\exp (u)-1$ and $u_{\text {exact }}=x y(1-x)(1-y)$.

$\mathrm{h}$ is the mesh grid

Here are some examples of results of the proposed algorithm corresponding to some tests functions

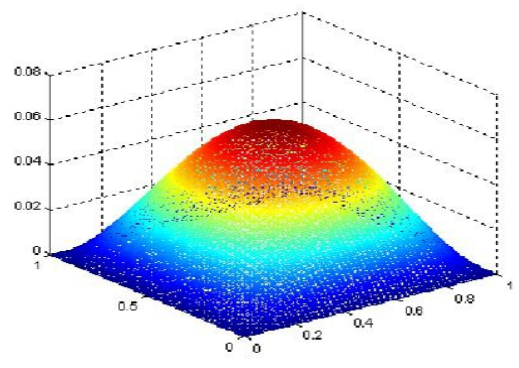

Figure 12: case1.

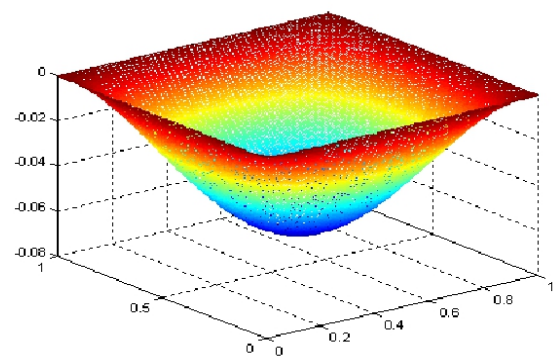

Figure 14: case3

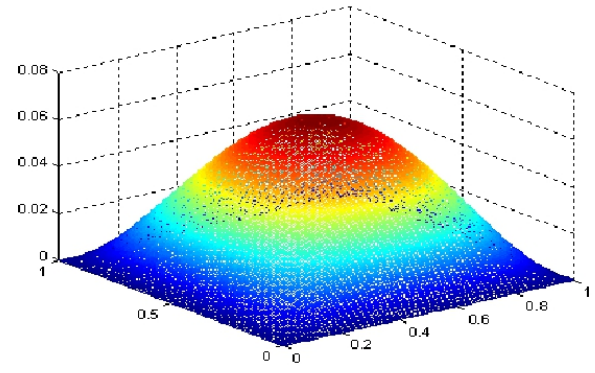

Figure 13: case2.

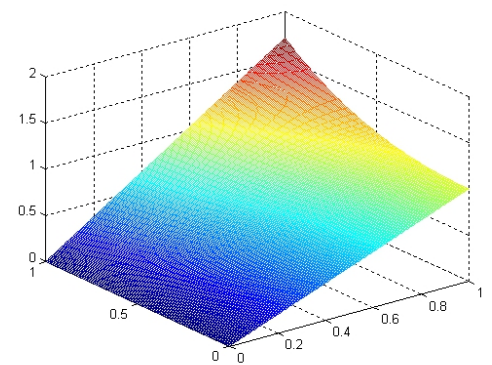

Figure 15: case4

case $1: \mathrm{u}=\mathrm{xy}(1-\mathrm{x})(1-\mathrm{y})$

case $2: u=\exp (x y(1-x)(1-y)-1)$.

case $3: u=\ln (1-x y(1-x)(1-y))$.

case $4: u=\exp \left(x^{*} y\right)-x y$

\section{Conclusion}

In this work we have developed an optimized domain decomposition algorithm applied to a non linear PDE. We firstly have proposed a proof of 
the convergence of the fixed point technique applied to the non linear equation. We have proposed a new approach for computing the convergence rate using the Fourier analysis and global optimization. Secondly we have presented several test-cases to show the efficiency of this approach. The fundamental result is that we obtained a well optimized rate of convergence of the proposed algorithm in comparison with global calculation using classical solvers. As perspective of the present work, we can study the following ideas:

- Generalize the method to non linear and non stationary equation

- Generalize the approach to the nonlinear diffusion equation.

- Apply the method to real problems in fluid dynamics, environmental sciences or the image processing.

\section{References:}

M.J.GAnDER(2006), Optimized Schwartz Methods, SIAM Journal on Numerical Analysis, 44(2), 699-731.

F.NATAF, F. ROGIER AND E. DE STURLER (1994), Optimal interface condition for domain decomposition methods, CMAP (Ecole Polytechnique).

D.K. KAUshiK, D.E. KeYES, B.F.SMith (1999, February), Newton-KrylovSchwarz Methods for Aerodynamics Problem: Compressible and uncompressible flows on unstructured grids. In Proceedings of the 11th International Conference on Domain Decomposition Methods. Domain Decomposition Press, Bergen.

Filipa CAetano, LAurence HalPern (2009), Algorithme de décomposition de domaine pour une équation de réaction-diffusion non linéaire, Abstract ,Université Paris 13.

Filipa CAetano, LAurenc Halpern, MARtin J. GANDER, Jeremie SZEFTEL(2010), Schwartz waveform relaxation algorithms for semilinear reaction-diffusion equations, 5(3), 487-505.

CAROLINE JAPHET(1998), Méthode de décomposition de domaine et conditions aux limites artificielles en mécanique des fluides : méthode optimisée d'ordre 2, (Doctoral dissertation, Université Paris-Nord-Paris XIII).

MOHAMED ZERIAB ES-SADEK(2009), Contribution á l'optimisation globale : Approche déterministe et stochastique et applications ; Doctoral dissertation, INSA de Rouen.

F.HECHT, Fluid Mechanics, Cours NSF03 , Master II, Mecanique, Parcours Fluid-Mechanics, Universite Pierre et Marie curie, Paris, France

Sofiane HadJI AND GouRI DHATT(1997), Assymptotic Newton method for solving incompressible flow, journal for numerical methods in fluids, 25(8), 861-878. 
Alfio Quarteroni, Alberto VAlli (1999), Domain decomposition methods for partial differential equations, Oxford science publications, May 27.

ANDREA ToSELli(2010), Domain Decomposition Methods - Algorithms and Theory, springer.

HAIM BREZIS(1994), Functional analysis, Dunod. 